\title{
To Optimize Fiber Lay Length in OPGW Cables Used in Power Transmission Networks
}

\author{
Faramarz E. Seraji, Ehsan Chaghazardi, Ali Emami \\ Optical Commun. Gr., Commun. Dept., Iran Telecom Research Center, Tehran, Iran \\ Corresponding author: feseraji@itrc.ac.ir.
}

\begin{abstract}
In this paper, the optimal fiber length in optical ground wire (OPGW) cable during production process is determined. The results show that in OPGW cable, if the fiber stranding length is less than the maximum lay length, the ultimate tensile stress (UTS) percentage decreases, but if it is higher, it would increase the bending losses and uses more fiber length, which is economically not viable. The calculation results show that by determining the maximum fiber lay length, the UTS percentage of OPGW cable can be controlled for a desired value.
\end{abstract}

Keyword: OPGW cable, fiber stranding length, optimal fiber loose tube.

\section{Introduction}

Optical Power Overhead Ground Wire (OPGW) fiber optic cables have different structures, whose type depends on the applications $[1,2]$. Use of power transmission tower is one of the immune ways of OPGW cable installation, where beside playing the role of ground wire is also used as a transmission medium [3]. The optical fiber used to make the OPGW cable is placed in a protective loose tube where the fiber can be moved freely.

The free movement of the optical fibers in this protective tube, which is wrapped around the center of the tube with a lay length of $\lambda$, is the basis of the work. Due to factors affecting the length of the cable, such as increasing temperature or tension, position of the fiber inside the protective tube will be set to change. In the case of increasing or decreasing the length of cable, the lay length $\lambda$ would increase or decrease, respectively.

Determining the lay length of optical fiber in the cable, which ultimately determines the length of fiber used, is important from the point of view of production economy, leading to huge production savings. In this paper, based on ultimate tensile stress (UTS), the fiber length and fiber stranding length in OPGW cable are optimally determined.

\section{Formulation of Analytical Relations}

The maximum tensile load of the optical fiber cable is equal to 0.9 times the maximum load $\left(f_{i}\right)$ that each of the wires in the cable fabrication can withstand and is determined by $F_{\text {break }}=0.9 \sum f_{i}$ [4]. In OPGW cable, the fiber is woven with a gauge (GA), aluminum alloy (AA), aluminum clad steel wires (ACS), aluminum (AL) wires based on the applications [5]. For each strand of wire, the value of $f_{i}$ is calculated from the relation $f_{i}=\sigma_{i} S_{i}$, where $\sigma_{i}$ is the tensile stress and $S_{i}$ denotes the cross-section of the wire [4]. In the design of aerial fiber optic cable OPGW, conditions such as wind pressure, cable length and weight, weight of snow and ice layers and distance between two power towers as well as physical conditions of crosssection, lay length, weight of wires, tensile and modulus of elasticity of wires not only determine the maximum load, but also have many effects on the optical and mechanical properties of the optical fiber, which in turn could disrupt the optical communication links. 
Therefore, according to these conditions, in selecting the common wire properties, the optical fiber requirements must also be considered, so that it will not be damaged. The presence of optical fiber in the cable also affects and limits the maximum tensile load. In OPGW cable, the tensile stress is the force per unit area, and the percentage of this force that the fibers in the cable bear before breakage will determine UTS [5].

The cable modulus of elasticity $E_{i}$ is obtained from the relation $E=\left(\sum E_{i} S_{i}\right) / S$, where $S_{i}$ denotes the cross-section of each wire and $S$ represents the cross-section of the whole cable. From the two relations $\sigma=E \varepsilon$ and $F=\sigma S$, the cable strain $\varepsilon=F / S E$ is obtained [5].

\subsection{Determination of the optimal fiber length in OPGW cable}

The optimal fiber length in the stainless steel loose tube (SSLT) in the OPGW cable is calculated by considering the percentage of UTS. The length of the fibers inside the SSLT tube are longer than the length of the tube by $\Delta L$. In fact, the fibers are stranded into the tube with a lay length $\lambda$. If $\Delta L$ is equal to the increase in cable length, the cable can withstand up to $100 \%$ of the UTS value, we have $L^{\prime}=\varepsilon L=(L F / S E)$, where $F$ is the value of UTS or RTS (rate of tensile stress), and $E$ and $S$ are defined as above.

By calculating the cable strain and the percentage of UTS requested by a customer, the lay length of the fiber inside the tube can be determined with the most optimal value. Because shorter the lay length, the more optical fiber length will be used in the cable and the more fiber standing inside the tube, resulting more bending, thus higher transmission losses [6]. From an economic point of view, when more optical fiber length is used, the volume of raw materials used in cable fabrication would increase, consequently, causing higher production costs. Therefore, choosing the maximum value $\lambda_{\max }$ of the lay length would present the optimal value for the optical fiber stranded inside the loose tube [7].

Figure 1 shows a part of an OPGW fiber cable in which a fiber of diameter $d$ is stranded around a core wire of diameter $D$ with a radius $R$ and length of $L$ with a step $\lambda$ [1].

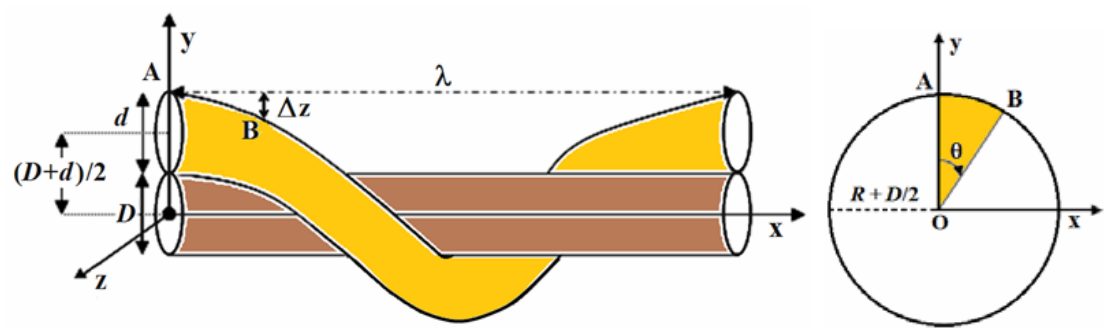

Fig. 1: The length of an optical fiber of diameter $d$ wrapped around a core wire of diameter $D$, radius $R$, and length $L$ with lay length $\lambda$.

For two points $A=\left(x_{A}, y_{A}, z_{A}\right)$ and $B=\left(x_{B}, y_{B}, z_{B}\right)$, as shown in Fig. 1, the following expressions can be derived as:

$$
A B=\sqrt{\left(x_{A}-x_{B}\right)^{2}+\left(y_{A}-y_{B}\right)^{2}+\left(z_{A}-z_{B}\right)^{2}}
$$

With reference to the geometry of Fig. 1, we can derive

$x=a \sin (\theta), y=a \cos (\theta)$

where $a=(D+d) / 2$.

By placing Eq. (2) in Eq. (1) and after simplification we will have: 


$$
A B=\sqrt{2 a^{2}\left[1-\cos \left(\Delta \theta_{A B}\right)\right]+\Delta Z^{2}}
$$

where $\Delta \theta_{A B}=(2 \pi / \lambda) \Delta Z$.

The length of the stranded optical fiber is obtained in one step from the following equation:

$\lambda^{\prime}=\lambda+\Delta \lambda=\lim _{\Delta Z \rightarrow 0}(\lambda / \Delta Z) A B$

If we define the tensile force $F$ as follows:

$F=\lim _{\Delta Z \rightarrow 0} \frac{1}{\Delta Z^{2}}[1-\cos ((2 \pi / \lambda) \Delta Z)]$,

then by using the d'Hospital rule, from Eq. (5) we can write:

$F=\frac{2 \pi^{2}}{\lambda^{2}}$

Therefore, Eq. (4) reduces to:

$$
\begin{aligned}
\lambda^{\prime} & =\lim _{\Delta Z \rightarrow 0} \lambda \sqrt{\frac{2 a^{2}}{\Delta z^{2}}[1-\cos ((2 \pi / \lambda) \Delta Z)]+1} \\
& =\lambda \sqrt{2 a^{2} F+1}
\end{aligned}
$$

By placing Eq. (6) in Eq. (7), we can write:

$$
\lambda^{\prime}=\sqrt{4 a^{2} \pi^{2}+\lambda^{2}}
$$

As a result, a change in the lay length of the optical fiber stranding inside the cable is obtained as follows:

$$
\Delta \lambda=\lambda^{\prime}-\lambda=\sqrt{4 a^{2} \pi^{2}+\lambda^{2}}-\lambda
$$

Since the protective loose tube can be divided into $n=L_{c} / \lambda$ segments, thus according to Fig. 2, the difference between the free length of the fiber and the protective tube $\Delta L_{F}=n \Delta \lambda$ is obtained as follows:

$$
\Delta L_{F}=\frac{L_{c}}{\lambda}\left(\sqrt{4[(D+d) / 2]^{2} \pi^{2}+\lambda^{2}}-\lambda\right)
$$

where the incremental length $\Delta L_{\mathrm{F}}$ should be equal to the maximum increase in fiber cable length at the time of stretching so that the minimum fiber length is used in the cable without breakage.
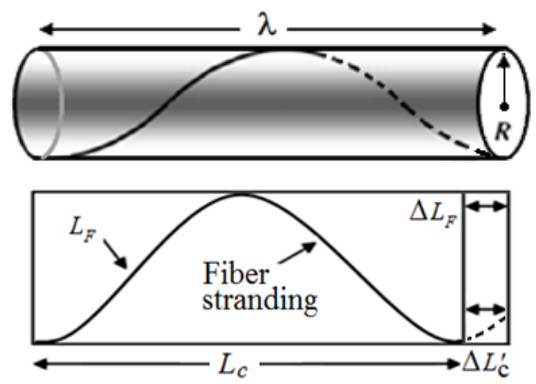

Fig. 2: Increase of cable and fiber lengths after stretching. 


\section{Numerical Result}

Based on the definition, the percentage of UTS can be calculated from the following relation in terms of lay length $\lambda[8]$ :

$$
\operatorname{UTS}(\%)=(S E / F)\left[\sqrt{\left[4(a / \lambda)^{2} \pi^{2}+1\right]}-1\right] \times 100
$$

If $\Delta L_{\mathrm{F}}$ is equal to the increase in the length of the cable, i.e., $\Delta L_{c}^{\prime}=\varepsilon L_{C}=\left(L_{c} F / S E\right)$, the fiber cable can withstand up to $100 \%$ of the UTS value. By calculating the cable strain and the percentage of UTS requested by the customer, the maximum lay length $\lambda_{\max }$ of the fiber inside the loose tube can be determined. As mentioned earlier, the shorter the lay length $\lambda$, would lead to increase of the total losses in the OPGW fiber cable. Under this condition, which is associated with an increase in the length of optical fiber consumption, will results in uneconomical production of OPGW fiber cables [5].

For example, we consider a typical standard OPGW fiber cable with the specifications mentioned in Fig. 3, having an outer diameter of $10.5 \mathrm{~mm}$, ACS wire diameter of $3.5 \mathrm{~mm}$ [8], and containing 5 optical fibers pairs. For this OPGW fiber cable with modulus of elasticity of $E=160 \mathrm{GPa}$ and maximum tensile load of $F=68.1 \mathrm{KN}$, the exponential changes in UTS percentage in terms of lay length $\lambda$ of the optical fiber in the cable are plotted in Fig. 4.

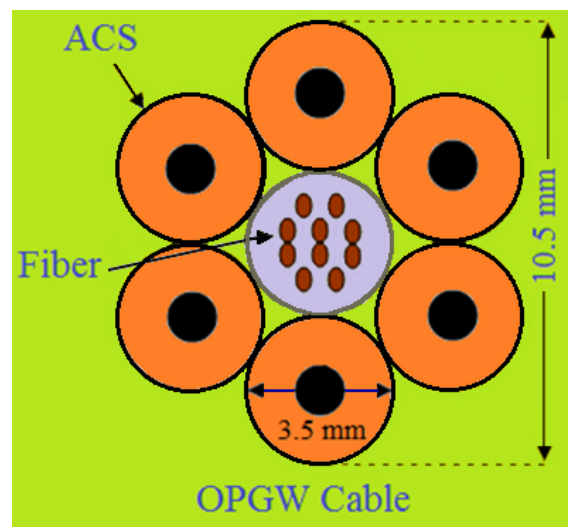

Fig. 3: Structure of a typical standard OPGW cable.

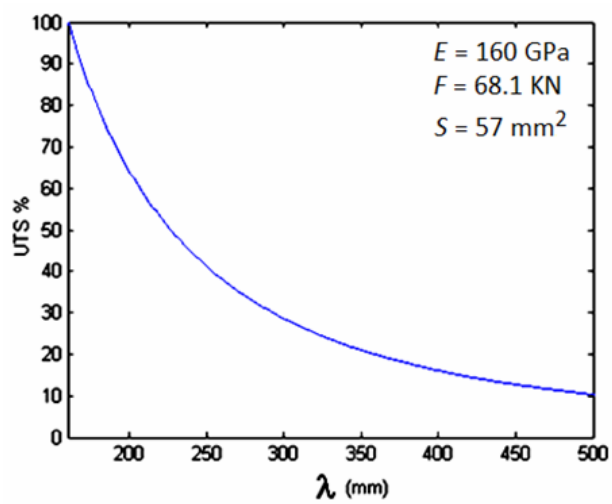

Fig. 4: UTS\% versus lay length $\lambda$ of optical fiber in the OPGW fiber cable. 


\section{Conclusion}

In this paper, the optimal fiber length in OPGW cable with lay length $\lambda$ is determined. If the fiber lay length is less than the maximum lay length $\lambda_{\max }$, the UTS percentage decreases, and if it is more than that, the bending losses would increase as the number of fiber stranding loops increase, resulting in more use of fiber length, which is not economically viable. By presenting the lay length maximization curve, it was shown that by calculating $\lambda_{\max }$ of the fiber in the cable, the UTS percentage of the OPGW cable can be controlled for a desired value.

\section{Acknowledgement}

The authors are grateful to the optical group leader of Communication Dept. of Iran Telecom Research Center for the project coordination.

\section{References}

[1] H. Amiri and F. E. Seraji, "Optimization of manufacturing parameters of optical fiber cables", Proc. CAOL 2005, 12-17 Sept., 2005, Yalta, Crimea, Ukraine.

[2] P. R. Bank and D. O. Lawrence, "Emerging standard in fiber optic telecommunication cables", Proc. SPIE, 224, pp. 149-158, 1980.

[3] IEEE standard construction of composite fiber optic overhead ground wire (OPGW) for use on electric utility power lines, IEEE Inc., Sept. 2005.

[4] Standard specification for concentric-lay-stranded Aluminum-clad steel conductors (designation: b416-98), ASTM International, Apr. 2006.

[5] IEEE standard construction of composite fiber optic overhead ground wire (OPGW) for use on electric utility power lines, IEEE Std. 1138-1994.

[6] Josep Martin Regalado, Alain Bertaina, and Lionel Provost, "Low Attenuation and High Excess-Fiber Length OPGW using G.652D bend insensitive fibers", International Wire \& Cable Symposium, Proc. the 61st IWCS Conference, pp.329-334., 2012.

[7] Syed Abrar Ali, Bilal Ahmad Alvi and Muhammad Asif, "OPGW - Our Experience in KESC”, 2008 IEEE Canada Electric Power Conference, 6-7 Oct. 2008, Vancouver, BC, Canada, pp. 1-6, 2008. DOI: 10.1109/EPC.2008.4763296.

[8] Standard specification for Concentric-Lay-Stranded Aluminum-Clad Steel Conductors, ASTM International, Apr. 2006 